\title{
OPEN Benefits and risks of antiplatelet medication in hemodynamically stable adult moyamoya disease
}

\author{
Chang Hwan Pang, Won-Sang Cho ${ }^{\bowtie}$, Hyun-Seung Kang \& Jeong Eun Kim
}

Revascularization surgery is considered a standard treatment for preventing additional stroke in symptomatic moyamoya disease (MMD). In hemodynamically stable, and asymptomatic or mildly symptomatic patients, however, the treatment strategy is controversial because of the obscure natural course of them. The authors analyzed the benefits and risks of antiplatelet medication in those patients. Medical data were retrospectively reviewed in 439 hemispheres of 243 patients with stable hemodynamic status. Overall, 121 patients (49.8\%) with 222 studied hemispheres $(50.6 \%)$ took antiplatelet medication. Symptomatic cerebral infarction and hemorrhage occurred in $10(2.3 \%)$ and $30(6.8 \%)$ hemispheres, over a mean follow-up of $62.0 \pm 43.4$ months (range 6-218 months). The use of antiplatelet agents was statistically insignificant in terms of symptomatic infarction, hemorrhage and improvement of ischemic symptoms. In subgroup analyses within the antiplatelet group according to drug potency and duration of medication, a longer duration of antiplatelet medication significantly improved ischemic symptoms (adjusted OR $1.02 ; 95 \% \mathrm{Cl} 1.01-1.03 ; \mathrm{p}=0.006$ ). Antiplatelet medication failed to prevent symptomatic cerebral infarction or improve ischemic symptoms. However, antiplatelet therapy did not increase the risk of cerebral hemorrhage.

Moyamoya disease (MMD) is an idiopathic progressive steno-occlusive cerebrovascular disease ${ }^{1}$. Hemodynamic insufficiency is a major pathophysiology in MMD, and microembolism is considered one of the causes of ischemic stroke ${ }^{1-6}$. Common clinical manifestations include cerebral ischemia in $50-76 \%$ of the patients and hemorrhage in $5-40 \%^{2,7-9}$. Revascularization surgery is accepted as an effective treatment to prevent additional stroke in symptomatic patients with ischemic and hemorrhagic $\mathrm{MMD}^{10,11}$. In hemodynamically stable adult MMD, optimal management considering perioperative complications and the natural course of the disease is still controversial. For patients with atherosclerotic cerebrovascular diseases ${ }^{12}$, antiplatelet agents have long been used, but there are few studies on the effect or risk of antiplatelet medication in $\mathrm{MMD}^{13-16}$. The authors performed a retrospective study to determine whether antiplatelet agents were useful to prevent symptomatic cerebral infarction and to improve ischemic symptoms and whether those agents were safe and caused no cerebral hemorrhage in hemodynamically stable adult MMD.

\section{Methods}

Patient selection. Medical data of the patients diagnosed with adult MMD between January 2003 and June 2018 were retrospectively reviewed under the approval of the institutional review board and the patient's informed consent was waived. Inclusion criteria were as follows: (1) age $\geq 20$ years; (2) compatibility with the diagnostic guidelines of the $\mathrm{MMDs}^{4,5,7}$; (3) an initial clinical follow-up without surgery of at least 6 months or more; (4) initially mild or fixed symptoms related to hemorrhage and ischemia, or asymptomatic presentation; (5) hemodynamically stable status on single-photon-emission computed tomography (SPECT); and (6) medical records pertaining to the evaluation of certain concomitant medical conditions at the first visit and during the follow-up period. All the patients were initially evaluated with SPECT, magnetic resonance imaging and/ or digital subtraction angiography. Hemodynamically stable status was defined as a normal or mild decrease in basal perfusion and a decrease in the reserve capacity after acetazolamide challenge at less than $50 \%$ of the basal perfusion on basal and acetazolamide-challenged SPECT with $99 \mathrm{mTc}$-hexamethylpropyleneamine oxime ${ }^{10}$. SPECT and/or magnetic resonance perfusion imaging with arterial spin labelling and fluid attenuated inversion recovery are usually followed-up every 2 years during the follow-up, and whenever ischemic symptoms and signs are aggravated. 


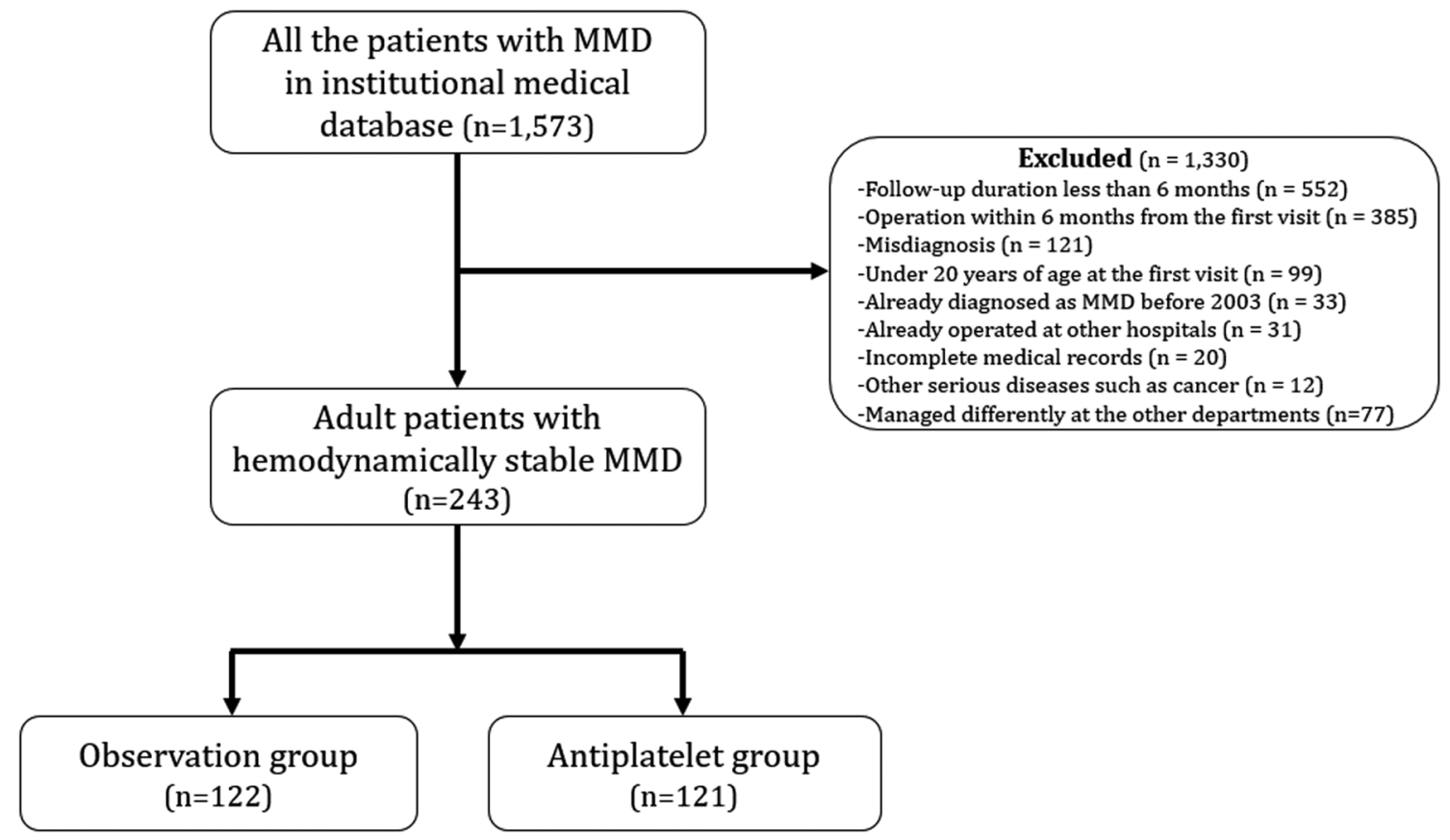

Figure 1. Flow chart of the patient selection. MMD moyamoya disease.

\begin{tabular}{|c|c|c|c|c|}
\hline Variables & Total & Observation group & Antiplatelet group & p value \\
\hline Patients/hemispheres, n (\%) & $243 / 439$ & $122(50.2) / 217(49.4)$ & $121(49.8) / 222(50.6)$ & \\
\hline Female, n (\%) & $182(74.9)$ & $94(77.0)$ & $88(72.7)$ & 0.437 \\
\hline Age, mean $\pm \mathrm{SD}$ (range), years & $43.7 \pm 11.4(20-74)$ & $43.3 \pm 10.7(20-67)$ & $44.2 \pm 12.0(20-74)$ & 0.541 \\
\hline Unilateral MMD, n (\%) & $47(19.3)$ & $27(22.1)$ & $20(16.5)$ & 0.269 \\
\hline Familial MMD, n (\%) & $47(19.3)$ & $25(20.5)$ & $22(18.2)$ & 0.649 \\
\hline Current smoking, n (\%) & $14(5.8)$ & $3(2.5)$ & $11(9.1)$ & 0.027 \\
\hline Hypertension, n (\%) & $85(35.0)$ & $36(29.5)$ & $49(40.5)$ & 0.073 \\
\hline Hyperlipidemia, n (\%) & $92(37.9)$ & $32(26.2)$ & 60 (49.6) & $<0.001$ \\
\hline \multicolumn{5}{|l|}{ Initial presentation, $\mathrm{n}^{\mathrm{a}}(\%)$} \\
\hline Cerebral ischemia & $198(45.1)$ & $88(40.6)$ & $110(49.5)$ & $0.058^{\dagger}$ \\
\hline Cerebral hemorrhage & $46(10.5)$ & $31(14.3)$ & $15(6.8)$ & $0.010^{\dagger}$ \\
\hline Incidental finding & $191(43.5)$ & $98(45.2)$ & $93(41.9)$ & $0.432^{\dagger}$ \\
\hline Other symptoms & $5(1.1)$ & 0 & $5(2.3)$ & $0.061^{\dagger}$ \\
\hline \multicolumn{5}{|c|}{ Perfusion status on SPECT, $\mathrm{n}^{\mathrm{a}}(\%)$} \\
\hline Decrease in basal perfusion & $173(39.4)$ & $79(36.4)$ & $94(42.3)$ & $0.203^{\dagger}$ \\
\hline Decrease in reserve capacity & $193(44.0)$ & $95(43.8)$ & $98(44.1)$ & $0.939^{\dagger}$ \\
\hline \multicolumn{5}{|l|}{ Existence of collaterals, $\mathbf{n}^{\mathbf{a}}(\%)$} \\
\hline Lenticulostriate & $125(28.5)$ & $71(32.7)$ & $54(24.3)$ & $0.051^{\dagger}$ \\
\hline Thalamic & 99 (22.6) & $53(24.4)$ & $46(20.7)$ & $0.353^{\dagger}$ \\
\hline Choroidal & $109(24.8)$ & $59(27.2)$ & $50(22.5)$ & $0.258^{\dagger}$ \\
\hline
\end{tabular}

Table 1. Baseline characteristics. $\mathrm{p}<0.05$ statistically significant (marked in bold). Unless specified otherwise, values indicate the number or data of patients. MMD moyamoya disease, SPECT single photon emission

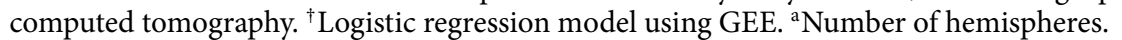

A total of 439 hemispheres of 243 patients were finally included (Fig. 1), consisting of 217 hemispheres in 122 patients (observation group) and 222 hemispheres in 121 patients (antiplatelet group). Baseline characteristics are presented in Table 1.

Antiplatelet medication. Antiplatelet drugs are not routinely prescribed for patients with MMD but are selectively used in this institution for patients presenting with acute cerebral infarction, with repeated ischemic 


\begin{tabular}{|c|c|c|c|c|}
\hline Variables & Total & Observation group & Antiplatelet group & p value \\
\hline \multicolumn{5}{|l|}{ Modified Rankin Scale, mean \pm SD (range) } \\
\hline Initial & $0.6 \pm 1.1(0-5)$ & $0.6 \pm 1.2(0-5)$ & $0.6 \pm 1.0(0-4)$ & 0.879 \\
\hline Last & $0.5 \pm 1.2(0-6)$ & $0.4 \pm 1.1(0-5)$ & $0.6 \pm 1.3(0-6)$ & 0.186 \\
\hline \multicolumn{5}{|l|}{ Symptomatic event during follow-up, $\mathrm{n}^{\mathrm{a}}(\%)$} \\
\hline Cerebral infarction & $10(2.3)$ & $5(2.3)$ & $5(2.3)$ & $1.000^{\dagger}$ \\
\hline Cerebral hemorrhage & $30(6.8)$ & $17(7.8)$ & $13(5.9)$ & $0.412^{\dagger}$ \\
\hline \multicolumn{5}{|l|}{ Change of ischemic symptoms, $\mathrm{n}^{\mathrm{a}}(\%)$} \\
\hline Improved & $63(31.8)$ & $29(33.0)$ & $34(30.9)$ & $0.099^{*}$ \\
\hline Unchanged & $80(40.4)$ & $41(46.6)$ & $39(35.5)$ & \\
\hline Aggravated & $55(27.8)$ & $18(20.5)$ & $37(33.6)$ & \\
\hline Follow-up duration, mean \pm SD (range), months & $62.0 \pm 43.4(6-218)$ & $51.5 \pm 35.0(6-177)$ & $72.5 \pm 48.4(7-218)$ & $<0.001$ \\
\hline
\end{tabular}

Table 2. Clinical results. $\mathrm{p}<0.05$ statistically significant (marked in bold). Unless specified otherwise, values indicate the data of patients. ${ }^{\dagger}$ Logistic regression model using GEE. ${ }^{\ddagger}$ Multinomial logistic regression model using GEE. ${ }^{a}$ Number of hemispheres.

symptoms despite stable hemodynamics ${ }^{3,5,6}$. Some patients with hemorrhagic and asymptomatic MMD to whom antiplatelet agents were prescribed for other medical problems were also included in this study.

Antiplatelet agents were classified into 4 types based on their relative potency. Potency 1 meant weak potency, such as for pentoxifylline, ibudilast and triflusal ${ }^{17-20}$. Potency 2 meant moderate potency, such as for acetyl salicylic acid, cilostazol, dipyridamole and sarpogrelate ${ }^{19,21,22}$. Potency 3 meant strong potency, including for clopidogrel and ticagrelor ${ }^{23,24}$. Finally, Potency 4 meant the strongest drug combinations, in which all the combinations were dual and including at least one drug of Potency $3^{25}$. For convenience, patients who did not take any antiplatelet agents during the follow-up included in this study were put into the Potency 0 group. Fortyfour (13.7\%) patients took antiplatelet agents of Potency 1, 171 (53.1\%) Potency 2, 68 (21.1\%) Potency 3 and 39 (12.1\%) Potency 4. Mean duration of antiplatelet drug medication was $72.5 \pm 48.4$ months (range7-218 months).

Clinical and radiological evaluation. The initial clinical presentation was divided into ischemic symptoms (transient ischemic attack [TIA] and cerebral infarction), cerebral hemorrhage (intracerebral, intraventricular, subdural and subarachnoid), and incidental findings (no symptoms or nonspecific symptoms such as headache and dizziness). The starting and ending neurological states were evaluated with the modified Rankin scale $^{26}$. Changes in the initial TIA symptoms were checked during the follow-up by evaluating the frequency and intensity of TIA symptoms and were classified into improved, unchanged and aggravated.

For the analysis of risk factors, some medical comorbidities were initially considered, including hypertension, dyslipidemia, diabetes mellitus, hypertension, heart disease, thyroid diseases, hematological diseases, familial history of MMD and current smoking. Among them, only a few factors were selected, and the other factors, due to their low incidence, were excluded to simplify the analysis. Patients who confirmed smoking during any of the follow-up period were defined as current smokers.

Transmedullary collaterals are well-known risk factors for cerebral hemorrhage and are usually composed of lenticulostriate, thalamic and choroidal collaterals ${ }^{27,28}$. When a collateral is dilated or extended beyond the normal territory, that collateral is defined as being present. All of them were evaluated on digital subtraction angiography.

Statistical analysis. Continuous variables are presented as mean \pm SD. Statistical analysis was performed using Student's $t$-test for continuous variables, while the $\chi^{2}$ test or Fisher's exact test was used for categorical variables. Logistic regression analysis was used to identify the causative variables of symptomatic infarction, symptomatic hemorrhage and improvement of ischemic symptoms. Fine and Gray's model was used to confirm whether antiplatelet medication affected cerebral infarction or hemorrhage. In addition, a robust sandwich covariance matrix estimate was used considering the correlated data when both hemispheres were evaluated. Since the duration of antiplatelet medication varied from patient to patient, it was considered a time-dependent covariate. To confirm the effects of antiplatelet agents on the improvement of TIA symptoms, multinomial logistic regression analysis was done using generalized estimating equations considering the associations of measurements between the hemispheres of each patient. Data are reported as ORs, HRs, 95\% CIs, and two-sided p values. $\mathrm{p}<0.050$ was considered significant. SAS statistical software (version 9.4; SAS Institute incorporation, Cary, North Carolina, USA) was used.

\section{Results}

Clinical results. Clinical results are summarized in Table 2. The clinical states in the two groups were similar at the first and last check-up periods. During the follow-up, symptomatic cerebral infarction and hemorrhage occurred in $10(2.3 \%)$ and 30 (6.8\%) hemispheres, respectively. Among 198 hemispheres initially presenting with ischemic symptoms, $72.2 \%(n=143)$ improved or remained unchanged during the mean follow-up of $62.0 \pm 43.4$ months (range 6-218 months). There were no differences between the two groups in terms of clinical status, symptomatic events or changes in ischemic symptoms, except the follow-up duration. 


\begin{tabular}{|l|l|l|l|}
\hline Variables & Adjusted HR & 95\% CI & p value \\
\hline Antiplatelet medication & 1.20 & $0.25-5.76$ & 0.835 \\
\hline Female & 2.12 & $0.29-15.68$ & 0.462 \\
\hline Age & 0.98 & $0.91-1.04$ & 0.437 \\
\hline Cerebral ischemia as initial presentation & 1.71 & $0.37-7.82$ & 0.491 \\
\hline Cerebral hemorrhage as initial presentation $^{\mathrm{a}}$ & NA & NA & NA \\
\hline Familial MMD & 1.74 & $0.42-7.24$ & 0.449 \\
\hline Current smoking & 3.17 & $0.21-46.36$ & 0.404 \\
\hline Hypertension & 2.71 & $0.57-12.93$ & 0.211 \\
\hline Hyperlipidemia & 1.14 & $0.33-3.88$ & 0.836 \\
\hline Decrease in basal perfusion on SPECT & 0.40 & $0.07-2.35$ & 0.310 \\
\hline Decrease in reserve capacity on SPECT & 0.66 & $0.19-2.38$ & 0.530 \\
\hline
\end{tabular}

Table 3. Logistic regression analysis of symptomatic cerebral infarction associated with antiplatelet medication during the follow-up. $\mathrm{p}<0.05$ statistically significant. NA not available, $M M D$ moyamoya disease, SPECT single photon emission computed tomography. ${ }^{a}$ Event number was 0 and not estimated.

\begin{tabular}{|l|l|l|l|}
\hline Variables & HR & $\mathbf{9 5 \%}$ CI & p value \\
\hline Antiplatelet medication & 0.56 & $0.24-1.34$ & 0.193 \\
\hline Female & 1.57 & $0.35-7.09$ & 0.556 \\
\hline Age & 1.01 & $0.98-1.04$ & 0.654 \\
\hline Cerebral ischemia as initial presentation & 0.57 & $0.20-1.62$ & 0.292 \\
\hline Cerebral hemorrhage as initial presentation & 0.86 & $0.27-2.72$ & 0.795 \\
\hline Familial MMD & 1.49 & $0.67-3.31$ & 0.332 \\
\hline Current smoking & 4.05 & $0.54-30.60$ & 0.176 \\
\hline Hypertension & 0.53 & $0.22-1.24$ & 0.141 \\
\hline Hyperlipidemia & 1.07 & $0.50-2.29$ & 0.857 \\
\hline Decrease in basal perfusion on SPECT & 1.22 & $0.58-2.60$ & 0.600 \\
\hline Decrease in reserve capacity on SPECT & 0.99 & $0.43-2.28$ & 0.981 \\
\hline Lenticulostriate collaterals & 1.42 & $0.57-3.54$ & 0.449 \\
\hline Thalamic collaterals & 1.52 & $0.63-3.65$ & 0.354 \\
\hline Choroidal collaterals & 2.84 & $1.31-6.18$ & $\mathbf{0 . 0 0 8}$ \\
\hline
\end{tabular}

Table 4. Logistic regression analysis of symptomatic cerebral hemorrhage associated with antiplatelet medication during the follow-up. $\mathrm{p}<0.05$ statistically significant (marked in bold). MMD moyamoya disease, SPECT single photon emission computed tomography.

Symptomatic cerebral infarction. A total of 10 symptomatic cerebral infarctions occurred during the follow-up, consisting of 5 events $(2.3 \%)$ in the observation group and $5(2.3 \%)$ in the antiplatelet group. Univariate analysis showed an insignificant effect of antiplatelet medication (unadjusted HR 1.16; 95\% CI 0.30-4.47; $\mathrm{p}=0.830$ ). In multivariate analysis, antiplatelet therapy failed to show a preventive effect on cerebral infarction (adjusted HR 1.17; 95\% CI 0.27-5.18; $\mathrm{p}=0.835$; Table 3).

Symptomatic cerebral hemorrhage. A total of 30 symptomatic cerebral hemorrhages occurred during the follow-up, consisting of 17 events (7.8\%) in the observation group and 13 (5.9\%) in the antiplatelet group. Univariate analysis showed a significant protective effect of antiplatelet medication on symptomatic cerebral hemorrhage (unadjusted HR: 0.44; 95\% CI 0.20-0.99; p=0.046). In multivariate analysis, however, antiplatelet therapy failed to show a preventive effect on cerebral hemorrhage (adjusted HR 1.19; 95\% CI 0.24-1.34; $\mathrm{p}=0.193$; Table 4). Interestingly, the existence of choroidal collaterals significantly increased the risk of cerebral hemorrhage (adjusted HR 2.84; 95\% CI 1.31-6.18; $\mathrm{p}=0.008$ ).

Improvement of ischemic symptoms. In a total of 198 hemispheres of 116 patients who initially presented with ischemic symptoms, 63 hemispheres (31.8\%) showed an improvement in ischemic symptoms. Antiplatelet medication failed to show a positive effect on the improvement of ischemic symptoms in the univariate (unadjusted OR $0.95 ; 95 \%$ CI $0.50-1.81 ; \mathrm{p}=0.876$ ) or multivariate analysis (adjusted OR 0.80; 95\% CI 0.40-1.62; $\mathrm{p}=0.541)($ Table 5).

Subgroup analysis within the antiplatelet group. In terms of symptomatic cerebral infarction, neither drug potency nor duration of medication had a preventive effect (Supplementary Tables 1 and 2). In terms of 


\begin{tabular}{|l|l|l|l|}
\hline Variables & Adjusted HR & 95\% CI & p value \\
\hline Antiplatelet medication & 0.80 & $0.40-1.62$ & 0.541 \\
\hline Female & 1.16 & $0.52-2.60$ & 0.711 \\
\hline Age & 1.01 & $0.97-1.04$ & 0.775 \\
\hline Familial MMD & 0.50 & $0.19-1.29$ & 0.150 \\
\hline Current smoking & 3.98 & $0.71-22.40$ & 0.117 \\
\hline Hypertension & 0.64 & $0.29-1.41$ & 0.270 \\
\hline Hyperlipidemia & 1.91 & $0.91-4.02$ & 0.089 \\
\hline Decrease in basal perfusion on SPECT & 1.72 & $0.89-3.33$ & 0.108 \\
\hline Decrease in reserve capacity on SPECT & 0.63 & $0.34-1.17$ & 0.144 \\
\hline
\end{tabular}

Table 5. Logistic regression analysis of improvement of ischemic symptoms associated with antiplatelet medication during the follow-up. $\mathrm{p}<0.05$ statistically significant. MMD moyamoya disease, SPECT single photon emission computed tomography.

symptomatic cerebral hemorrhage, the potency of antiplatelet agents was insignificant (Supplementary Table 3), and the duration of medication was of borderline significance (adjusted HR 0.99; 95\% CI 0.97-0.100; $p=0.085$ ). In the multivariate analysis of the duration of medication, however, cerebral ischemia at the initial presentation was a significant preventive factor against cerebral hemorrhage (adjusted HR 0.07; 95\% CI 0.01-0.77; $p=0.030$; Supplementary Table 4). Finally, in terms of the improvement of ischemic symptoms, a longer duration of antiplatelet medication (adjusted OR 1.02; 95\% CI 1.01-1.03; $\mathrm{p}=0.006$ ) and current smoking (adjusted OR 19.05; 95\% CI 1.27-286.87; $\mathrm{p}=0.033$ ) showed positive effects (Supplementary Table 5).

\section{Discussion}

Surgical revascularization is well known to be effective in preventing recurrent stroke in adult $\mathrm{MMD}^{4,10,11}$. In patients with hemodynamically stable but clinically symptomatic MMD, however, effective medical management has long been debated, weighing unignorable natural course against surgical burdens $\mathrm{s}^{5,8,10,11,13-16,29,30}$. Disappointingly, in this study, the use of antiplatelet agents had no significant effect in the prevention of cerebral infarction, improvement of initial ischemic symptoms or occurrence of cerebral hemorrhage. The existence of choroidal collaterals was a significant risk factor for cerebral hemorrhage, which has recently become well known ${ }^{27,28}$. In the subgroup analysis within the antiplatelet group, duration of antiplatelet medication and current smoking were significant factors improving the initial ischemic symptoms. The positive result of current smoking should be cautiously interpreted, considering the wide 95\% CI.

The natural history of MMD varies slightly according to the ethnicity, institution and status of the patient group. Patients with some characteristics, such as Asian ethnicity, symptomatic presentation and a low perfusion status, are reported to show a higher risk of stroke ${ }^{8,29,31-33}$, and these are usually considered surgical indications for the prevention of recurrent bleeding and infarction ${ }^{11,29}$. Asymptomatic or hemodynamically stable symptomatic patients seem to have a benign clinical course; however, they are not in a stable status, given their annual stroke risk of 3.2-4.5\% $\%^{8,33}$. Meanwhile, perioperative complications occur often (cerebral hemorrhage in $3.1 \%$; infarction in $4.0 \%$ ), so that at least 2 or 3 years are needed to overcome the natural course of disease in conservatively managed patients ${ }^{10,29,30}$. Therefore, physicians always feel a strong urge to find effective medical management for hemodynamically stable patients with asymptomatic or mildly symptomatic presentations.

Antiplatelet agents have long been used for primary and secondary prevention of stroke and are backed by concrete evidences $^{12}$. They are frequently prescribed for MMD patients presenting with ischemic symptoms in the real world ${ }^{34,35}$, and antiplatelet medication is recommended in the recent guidelines for MMD, although the level of evidence is not high ${ }^{5}$. The major pathomechanism of MMD is hemodynamic insufficiency caused by progressive steno-occlusion of the intracranial arteries ${ }^{1,2,4,5}$. However, as intraluminal microthromboembolism was recently shown to be one of the ischemic symptoms in patients with $\mathrm{MMD}^{3,6}$, the use of antiplatelet agents is increasing and is expected to inhibit platelet aggregation and thrombus formation. Moreover, cilostazol is thought to improve hemodynamic insufficiency through a vasodilatory effect as one of its pleiotropic effects ${ }^{15}$. Antiplatelet medication is selectively used in this institution for patients presenting with repeated ischemic symptoms and signs despite stable hemodynamics, a situation probably caused by microembolism. However, there still exists a major risk of antiplatelet agents to look into, intracranial hemorrhage ${ }^{21}$, though there is no definite evidence on this connection.

A few studies about the effects of antiplatelet medication have been recently reported in patients with $\mathrm{MMD}^{13-16}$. Their preventive effect on ischemic stroke is not consistent, though antiplatelet agents do not seem to increase the risk of cerebral hemorrhage. However, those studies have some limitations, such as not mentioning the kinds of drugs used or the indications of drug administration and the inhomogeneous basal statuses of the patients. A prospective observational study reported that antiplatelet agents did not reduce recurrent cerebral infarction, whereas the rate of cerebral hemorrhage was significantly lower in the antiplatelet group ${ }^{13}$. However, the results were derived from the univariate analysis within the ischemic subgroup alone. A retrospective propensity score-matched study demonstrated that patients who received prehospital antiplatelet agents had a significantly better initial status and outcome ${ }^{14}$. In a propensity score-matched study of national health data ${ }^{15}$, any use of antiplatelet agents reduced the mortality rate, and cilostazol showed the best effect. A recent propensity score-matched study in adult ischemic MMD showed that the risk of recurrent ischemic attack was lowest in the 
surgery group and that the antiplatelet group also had lower recurrence rates than the observation group, with no differences in bleeding events or functional outcomes ${ }^{16}$. However, treatment modality was determined only based on the patients' clinical states and their choice.

In this study, the use of antiplatelet agents did not influence the occurrence of cerebral infarction, hemorrhage and the improvement of initial ischemic symptoms. Patients visiting this medical team are evaluated according to the predetermined work-up protocol, and the treatment strategy is determined based on the test findings and indications this team has established ${ }^{4,8,10,36,37}$. Therefore, it is thought that the patients included in this study would be more homogeneous than those in previous reports. This study has some limitations, such as its retrospective nature, lack of randomization and use of a few kinds of antiplatelet agents ${ }^{13-16}$. Interestingly, in a search of the institutional database, the authors found not a few adult MMD patients who were managed by the other teams of the same institution. Analyzing of a total of 439 hemispheres in 243 patients in the same period, antiplatelet medication was insignificant in terms of cerebral infarction, hemorrhage and improvement of ischemic symptoms. In the subgroup analysis within the antiplatelet group of 110 hemispheres in 66 patients, however, a longer duration of antiplatelet medication significantly reduced symptomatic cerebral infarction and hemorrhage. Such different results are thought to originate from the inhomogeneous basal characteristics of the patients included in the additional analysis because the management strategies are very different among the medical teams. Thus, a study design with a clear definition of the patient group is very important for obtaining valuable results, and this should be considered when designing prospective studies in the near future. As revascularization surgery for hemodynamically unstable MMD is definitely effective in the prevention of recurrent events $^{10,13}$, surgery is judged to be more beneficial than antiplatelet medication in unstable patients despite the benefits of antiplatelet medication and the perioperative complications. In hemodynamically stable, and asymptomatic or mildly symptomatic patients, antiplatelet medication could be tried, considering the natural course of those patients and their conditions $s^{8,13}$, because the risk of antiplatelet agents seems insignificant and drugs are effective in certain patients. However, choroidal collaterals are a recent well-known risk factor of rebleeding and de novo bleeding in $\mathrm{MMD}^{27,28}$, which was also proved in this study. Although antiplatelet medication was insignificant in bleeding event in this study, it is necessary to be more careful in prescribing antiplatelet agents in patients with choroidal collaterals.

In conclusion, antiplatelet medication in adult patients with hemodynamically stable MMD did not show any benefits or risks in the occurrence of symptomatic stroke or improvement of initial ischemic symptoms, despite other significant factors related to cerebral hemorrhage or the improvement of ischemic symptoms in the subgroup analysis. Well-designed prospective studies with some drugs widely used and recently known to be effective, such as aspirin, clopidogrel and cilostazol ${ }^{15,38}$, are expected to overcome the limitations of previous studies, including this, and to suggest more concrete answers.

\section{Data availability}

The data are available from the corresponding author on reasonable request approved the institutional review boards of Seoul National University Hospital.

Received: 14 July 2021; Accepted: 17 September 2021

Published online: 29 September 2021

\section{References}

1. Suzuki, J. \& Takaku, A. Cerebrovascular, "moyamoya" disease. Disease showing abnormal net-like vessels in base of brain. Arch. Neurol. 20, 288-299. https://doi.org/10.1001/archneur.1969.00480090076012 (1969).

2. Kuroda, S. \& Houkin, K. Moyamoya disease: Current concepts and future perspectives. Lancet Neurol. 7, 1056-1066. https://doi. org/10.1016/S1474-4422(08)70240-0 (2008).

3. Jeon, C., Yeon, J. Y., Jo, K. I., Hong, S. C. \& Kim, J. S. Clinical role of microembolic signals in adult moyamoya disease with ischemic stroke. Stroke 50, 1130-1135. https://doi.org/10.1161/Strokeaha.118.022490 (2019).

4. Kim, J. E. \& Jeon, J. S. An update on the diagnosis and treatment of adult Moyamoya disease taking into consideration controversial issues. Neurol. Res. 36, 407-416. https://doi.org/10.1179/1743132814y.0000000351 (2014).

5. Research Committee on the Pathology, Treatment of Spontaneous Occlusion of the Circle of, W. \& Health Labour Sciences Research Grant for Research on Measures for Infractable, D. Guidelines for diagnosis and treatment of moyamoya disease (spontaneous occlusion of the circle of Willis). Neurol. Med. Chir. (Tokyo) 52, 245-266. https://doi.org/10.2176/nmc.52.245 (2012).

6. Kraemer, M., Heienbrok, W. \& Berlit, P. Moyamoya disease in Europeans. Stroke 39, 3193-3200. https://doi.org/10.1161/Strok eaha.107.513408 (2008).

7. Kim, J. E. et al. Clinical features of adult moyamoya disease with special reference to the diagnosis. Neurol. Med. Chir. (Tokyo) 52, 311-317. https://doi.org/10.2176/nmc.52.311 (2012).

8. Cho, W. S. et al. The natural clinical course of hemodynamically stable adult moyamoya disease. J. Neurosurg. 122, 82-89. https:// doi.org/10.3171/2014.9.Jns132281 (2015).

9. Scott, R. M. \& Smith, E. R. Medical progress moyamoya disease and moyamoya syndrome. N. Engl. J. Med. 360, 1226-1237. https:// doi.org/10.1056/NEJMra0804622 (2009).

10. Cho, W. S. et al. Long-term outcomes after combined revascularization surgery in adult moyamoya disease. Stroke 45 , 3025. https:// doi.org/10.1161/Strokeaha.114.005624 (2014).

11. Miyamoto, S. et al. Effects of extracranial-intracranial bypass for patients with hemorrhagic moyamoya disease results of the japan adult moyamoya trial. Stroke 45, 1415-1421. https://doi.org/10.1161/Strokeaha.113.004386 (2014).

12. Kernan, W. N. et al. Guidelines for the prevention of stroke in patients with stroke and transient ischemic attack: A guideline for healthcare professionals from the American Heart Association/American Stroke Association. Stroke 45, 2160-2236. https://doi. org/10.1161/STR.0000000000000024 (2014).

13. Yamada, S. et al. Effects of surgery and antiplatelet therapy in ten-year follow-up from the registry study of research committee on moyamoya disease in Japan. J. Stroke Cerebrovasc. 25, 340-349. https://doi.org/10.1016/j.jstrokecerebrovasdis.2015.10.003 (2016).

14. Onozuka, D. et al. Prehospital antiplatelet use and functional status on admission of patients with non-haemorrhagic moyamoya disease: A nationwide retrospective cohort study (J-ASPECT study). BMJ Open https://doi.org/10.1136/bmjopen-2015-009942 (2016). 
15. Seo, W. K. et al. Association of antiplatelet therapy, including cilostazol, with improved survival in patients with moyamoya disease in a nationwide study. J. Am. Heart Assoc. 10, e017701. https://doi.org/10.1161/JAHA.120.017701 (2021).

16. Ye, F. et al. Efficacy and safety of antiplatelet agents for adult patients with ischemic moyamoya disease. Front. Neurol. 11, 608000. https://doi.org/10.3389/fneur.2020.608000 (2020).

17. Chan, Y. W. \& Kay, C. S. Pentoxifylline in the treatment of acute ischaemic stroke-A reappraisal in Chinese stroke patients. Clin. Exp. Neurol. 30, 110-116 (1993).

18. Nishimura, H., Naritomi, H., Iwamoto, Y., Tachibana, H. \& Sugita, M. In vivo evaluation of antiplatelet agents in gerbil model of carotid artery thrombosis. Stroke 27, 1099-1103. https://doi.org/10.1161/01.str.27.6.1099 (1996) (discussion 1104).

19. Huang, H. P. et al. Comparative efficacy and safety of nine anti-platelet therapies for patients with ischemic stroke or transient ischemic attack: A mixed treatment comparisons. Mol. Neurobiol. 54, 1456-1466. https://doi.org/10.1007/s12035-016-9739-z (2017).

20. Matias-Guiu, J. et al. Comparison of triflusal and aspirin for prevention of vascular events in patients after cerebral infarction: the TACIP Study: A randomized, double-blind, multicenter trial. Stroke 34, 840-848. https://doi.org/10.1161/01.STR.0000063141. 24491.50 (2003).

21. Antithrombotic Trialists' Collaboration et al. Aspirin in the primary and secondary prevention of vascular disease: Collaborative meta-analysis of individual participant data from randomised trials. Lancet 373, 1849-1860. https://doi.org/10.1016/S01406736(09)60503-1 (2009).

22. Huang, Y. et al. Cilostazol as an alternative to aspirin after ischaemic stroke: A randomised, double-blind, pilot study. Lancet Neurol. 7, 494-499. https://doi.org/10.1016/S1474-4422(08)70094-2 (2008).

23. Sacco, R. L. et al. Aspirin and extended-release dipyridamole versus clopidogrel for recurrent stroke. N. Engl. J. Med. 359, 12381251. https://doi.org/10.1056/NEJMoa0805002 (2008).

24. Wang, Y. et al. Ticagrelor plus aspirin versus clopidogrel plus aspirin for platelet reactivity in patients with minor stroke or transient ischaemic attack: Open label, blinded endpoint, randomised controlled phase II trial. BMJ 365, 12211. https://doi.org/10.1136/ bmj.12211 (2019).

25. Hao, Q. et al. Clopidogrel plus aspirin versus aspirin alone for acute minor ischaemic stroke or high risk transient ischaemic attack: Systematic review and meta-analysis. BMJ 363, k5108. https://doi.org/10.1136/bmj.k5108 (2018).

26. Uyttenboogaart, M., Stewart, R. E., Vroomen, P. C., De Keyser, J. \& Luijckx, G. J. Optimizing cutoff scores for the Barthel index and the modified Rankin scale for defining outcome in acute stroke trials. Stroke 36, 1984-1987. https://doi.org/10.1161/01.STR. 0000177872.87960 .61 (2005).

27. Funaki, T. et al. High rebleeding risk associated with choroidal collateral vessels in hemorrhagic moyamoya disease: Analysis of a nonsurgical cohort in the Japan Adult Moyamoya Trial. J. Neurosurg. 130, 525-530. https://doi.org/10.3171/2017.9.Jns17576 (2019).

28. Funaki, T. et al. Effect of choroidal collateral vessels on de novo hemorrhage in moyamoya disease: Analysis of nonhemorrhagic hemispheres in the Japan Adult Moyamoya Trial. J. Neurosurg. 132, 408-414. https://doi.org/10.3171/2018.10.JNS181139 (2019).

29. Kim, T. et al. Stroke prevention by direct revascularization for patients with adult-onset moyamoya disease presenting with ischemia. J. Neurosurg. 124, 1788-1793. https://doi.org/10.3171/2015.6.Jns151105 (2016).

30. Sun, H. et al. Perioperative complications and long-term outcomes after bypasses in adults with moyamoya disease: A systematic review and meta-analysis. World Neurosurg. 92, 179-188. https://doi.org/10.1016/j.wneu.2016.04.083 (2016).

31. Herve, D. et al. Predictors of clinical or cerebral lesion progression in adult moyamoya angiopathy. Neurology 93, e388-e397. https://doi.org/10.1212/WNL.0000000000007819 (2019).

32. Liu, X. J. et al. Clinical features and long-term outcomes of moyamoya disease: A single-center experience with 528 cases in China. J. Neurosurg. 122, 392-399. https://doi.org/10.3171/2014.10.Jns132369 (2015).

33. Kuroda, S., Hashimoto, N., Yoshimoto, T. \& Iwasaki, Y. Radiological findings, clinical course, and outcome in asymptomatic Moyamoya disease-Results of multicenter survey in Japan. Stroke 38, 1430-1435. https://doi.org/10.1161/Strokeaha.106.478297 (2007).

34. Kraemer, M., Berlit, P., Diesner, F. \& Khan, N. What is the expert's option on antiplatelet therapy in moyamoya disease? Results of a worldwide Survey. Eur. J. Neurol. 19, 163-167. https://doi.org/10.1111/j.1468-1331.2011.03481.x (2012).

35. Oki, K. et al. Trends of antiplatelet therapy for the management of moyamoya disease in Japan: Results of a nationwide survey. J. Stroke Cerebrovasc. 27, 3605-3612. https://doi.org/10.1016/j.jstrokecerebrovasdis.2018.08.030 (2018).

36. Cho, W. S. et al. Can combined bypass surgery at middle cerebral artery territory save anterior cerebral artery territory in adult moyamoya disease?. Neurosurgery 80, 431-437. https://doi.org/10.1227/Neu.0000000000001354 (2017).

37. Kim, J. E. \& Pang, C. H. Diagnosis and treatment of adult Moyamoya disease. J. Korean Med. Assoc. 62, 577-585. https://doi.org/ 10.5124/jkma.2019.62.11.577 (2019).

38. Ando, S. et al. Cilostazol may improve cognition better than clopidogrel in non-surgical adult patients with ischemic moyamoya disease: Subanalysis of a prospective cohort. Neurol. Res. 41, 480-487. https://doi.org/10.1080/01616412.2019.1580455 (2019).

\section{Acknowledgements}

The statistical analysis of this paper was conducted by Medical Research Collaborating Center of Seoul National University Hospital Biomedical Research Institute.

\section{Author contributions}

W.S.C. developed the study concept and design. W.S.C and C.H.P. collected, interpreted the data and drafted the manuscript and prepared figure. W.S.C., J.E.K. and H.S.K. revised the drafted manuscript. All authors reviewed the manuscript.

\section{Competing interests}

This work was supported by a grant of the Korea Health Technology R\&D Project through the Korea Health Industry Development Institute (KHIDI) funded by the Ministry of Health \& Welfare, Republic of Korea (Grant number : HI17C1561). The authors declare no conflict of interest concerning the materials or methods used in this study or the findings specified in this paper. No benefits of any form have been or will be received from any commercial party directly or indirectly related to the subject of this paper.

\section{Additional information}

Supplementary Information The online version contains supplementary material available at https://doi.org/ 10.1038/s41598-021-99009-1.

Correspondence and requests for materials should be addressed to W.-S.C. 
Reprints and permissions information is available at www.nature.com/reprints.

Publisher's note Springer Nature remains neutral with regard to jurisdictional claims in published maps and institutional affiliations.

(c) (i) Open Access This article is licensed under a Creative Commons Attribution 4.0 International License, which permits use, sharing, adaptation, distribution and reproduction in any medium or format, as long as you give appropriate credit to the original author(s) and the source, provide a link to the Creative Commons licence, and indicate if changes were made. The images or other third party material in this article are included in the article's Creative Commons licence, unless indicated otherwise in a credit line to the material. If material is not included in the article's Creative Commons licence and your intended use is not permitted by statutory regulation or exceeds the permitted use, you will need to obtain permission directly from the copyright holder. To view a copy of this licence, visit http://creativecommons.org/licenses/by/4.0/.

(C) The Author(s) 2021 